\title{
Indoor Localization System Using Wireless Sensor Network
}

\author{
Ekhlas K. Hamza ${ }^{1}$, Hanan Hussain Al-asady ${ }^{2}$ \\ ${ }^{1,2}$ Control and Systems Engineering Department, University of Technology, Baghdad \\ 1drekhladkadhum@gmail.com, ${ }^{2}$ aboala8834598@yahoo.com
}

\begin{abstract}
Building a precise low cost indoor positioning and navigation wireless system is a challenging task. The accuracy and cost should be taken together into account. Especially, when we need a system to be built in a harsh environment. In recent years, several researches have been implemented to build different indoor positioning system (IPS) types for human movement using wireless commercial sensors. The aim of this paper is to prove that it is not always the case that having a larger number of anchor nodes will increase the accuracy. Two and three anchor nodes of ultra-wide band with or without the commercial devices (DW 1000) could be implemented in this work to find the Localization of objects in different indoor positioning system, for which the results showed that sometimes three anchor nodes are better than two and vice versa. It depends on how to install the anchor nodes in an appropriate scenario that may allow utilizing a smaller number of anchors while maintaining the required accuracy and cost.
\end{abstract}

Index Terms - Wireless systems, wireless sensors network, indoor positioning system, ultra-wide band signal.

\section{INTRODUCTION}

Nowadays, the wireless indoor localization is an interesting topic in many applications [1]. Indoor positioning systems aim for providing a precise position estimate inside buildings, which is a particularly tricky task, due to phenomena such as non-line-of-sight and multipath, caused by walls and other obstacles. In other words, the positioning systems based on distances for industrial and mobile robot applications require the position of anchors in advance. With known anchor positions, a tag or device (e.g. a mobile robot or smartphone) can determine its position based on distance measurement with respect to anchors. This method is called alteration [2]. In particular, time-based positioning techniques rely on inter-nodes distance estimates evaluated from the time of flight of signals traveling between pairs of nodes. Given the distance estimates between few nodes, denoted as Anchor Nodes (ANs), and a Target Node (TN), the TN's position can be estimated.

Among the wide variety of localization, localization systems with an accuracy better than 1 meter for human movement localization system are the key enabler for providing position specific information on mobile devices in buildings [3]. It measures the distance between a point of an unknown position (mobile $\mathrm{M}$ ) and three points at known positions [2]. In practice, the propagation time of electromagnetic wave is measured and the distance is calculated by multiplication with the velocity of light. Measuring propagation times requires the knowledge of the starting time at the transmitter and the time of arrival (TOA) at the receiver with clocks to be synchronized [4].

It is possible to omit the start time measurement introducing an additional receiver at a known position and measuring the time differences of arrival (TDOA) at the four receivers of a signal transmitted by the mobile terminal [3]. According to Shannon's law, capacity and hence data rate 
increases linearly with bandwidth, which makes the ultra-wide band (UWB) technology a very promising future candidate for the transmission of ultra-high data rates. Up to now, only few UWB systems are commercially available, and ultra-wideband transmission is still a topic of research [2].

The rest of the article is structured as follows: In Section 2 we present theoretical analysis of UWB ranging and Section 3 discusses our proposal: Indoor Propagation Channel types and their properties. In Section 4 we evaluate the methodology with simulation data as well as real data. Finally, we summarize our work in Section 5 .

\section{THEORICAL ANALYSIS OF UWB TECHNOLOGY [5]}

A classical time of arrival based method is called two-way ranging (TWR). The practical ranging demonstration is described in Fig.1. The leader observes a round trip time LRT $=$ TRR - TSB and a turnaround time LTA $=$ TSF - TRR, where TSB, TRR and TSF are the leader send-time, receive -time and future send -time, respectively. The value of the transmission time $\mathrm{T}$ is computed at both leader ( $\mathrm{Tl}$ ) and follower ( $\mathrm{Tf}$ ) as:

$$
\begin{aligned}
& 2 T l=(T R R-T S B)-(T S R-T R B) \quad 2.1 \\
& 2 T f=(T R F-T S R)-(T S F-T R R) \quad 2.2
\end{aligned}
$$

The result is then divided by 2 to get one way trip time.

$$
T=2 T l+2 T f / 2 \times 2
$$

Thus the distance between the two prototypes is:

$$
d=T \times C
$$

Here $\mathrm{Cq}$ is the speed of light.

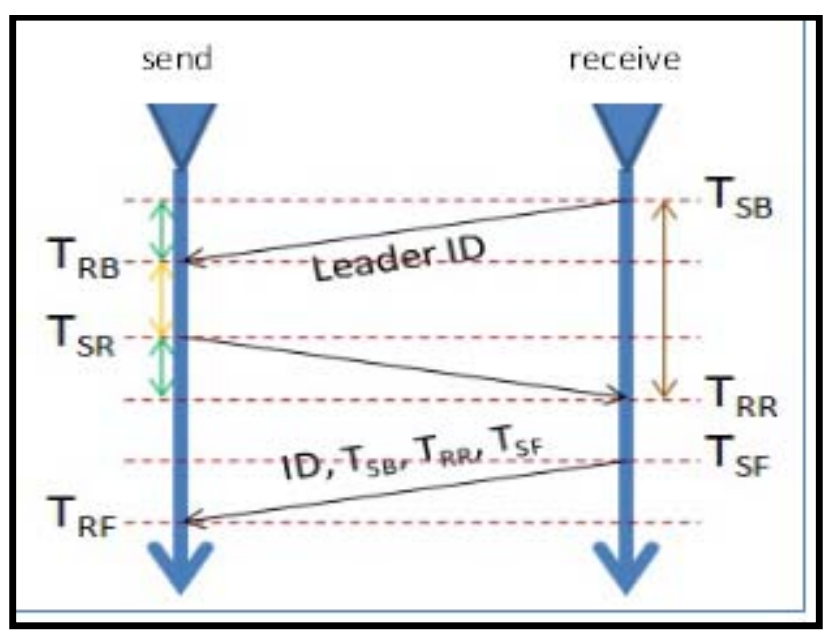

Fig. 1. TW-RAnging Method From [5]

\section{INDOOR CHANNEL TYPES}

When considering a channel between a transmitter and a receiver in a radio scheme one of the most important properties of the channel is whether it is either [6]: -

\section{A. Line of Sight (LOS)}

An optically clear line of sight between two wireless nodes exists if an imaginary straight line can be drawn between the antennas of the two nodes. It is important to make the distinction between an optically clear line of sight and clear line of sight from an RF perspective [6]: -

a) An optically clear LOS exists when no physical objects obstruct viewing one antenna from the location of the other antenna [7]. 
b) An RF clear line of sight exists if a defined area around the optical line of sight, known as the Fresnel Zone, is clear of obstacles [7].

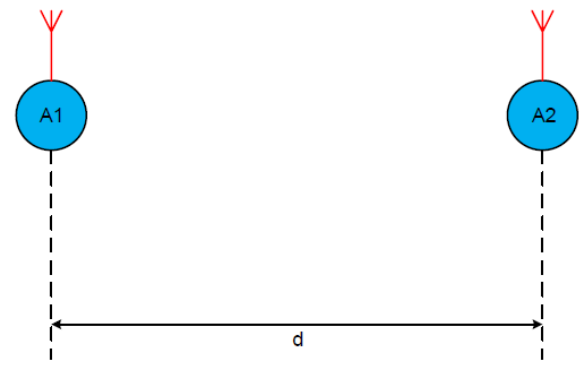

Fig. 2. Line OF Sight CHANNEL FROM [7]

$$
d^{\prime}=c * T O F
$$

Where [7]: -

o TOF is the time of flight between the two nodes

o d is the actual physical distance between the two nodes in meters

o d' is the calculated distance between the two nodes in meters

$\mathrm{o} \quad \mathrm{c}$ is the speed of light in $\mathrm{m} / \mathrm{s}$
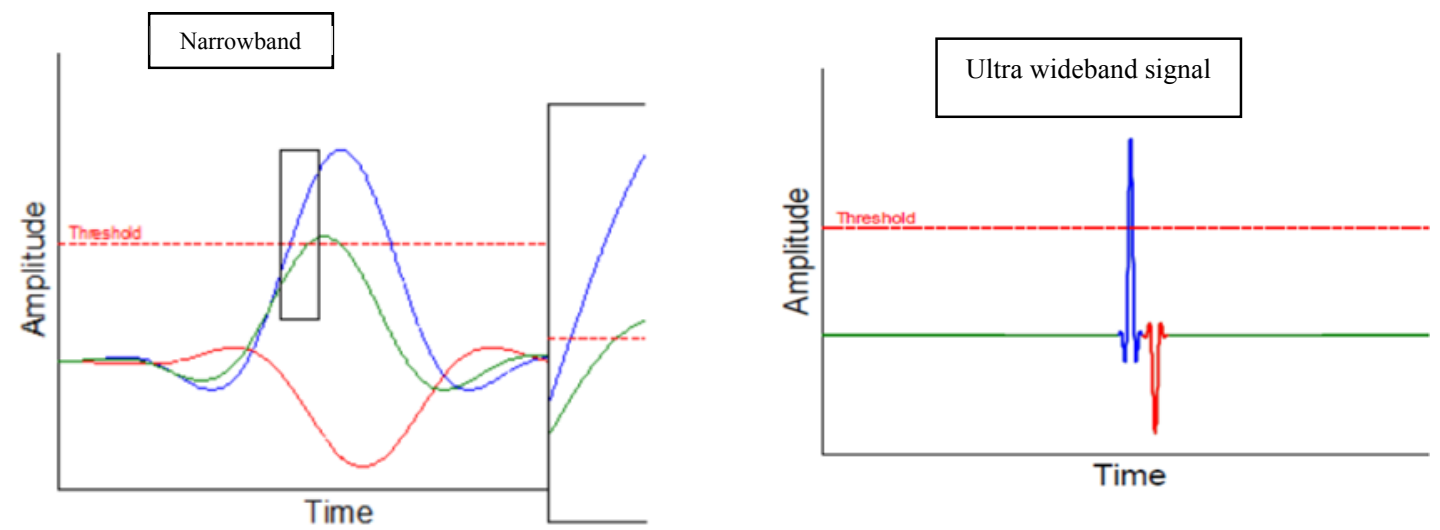

Fig. 3. THE DifFERENCE BETWEEN NARROWBAND SIGNAL AND UltRA WIDEBAND SIGNAL WITH REFLECTION FROM [7].

\section{B. Non Line of Sight (NLOS )[7]}

There are three aspects to consider when examining NLOS operation: -

a) Reduction in communications range due to overall signal attenuation

b) Reduction in direct path detection range due to attenuation of the direct path signal

c) Time of Flight errors due to differences in the refractive index of the obstructing material 


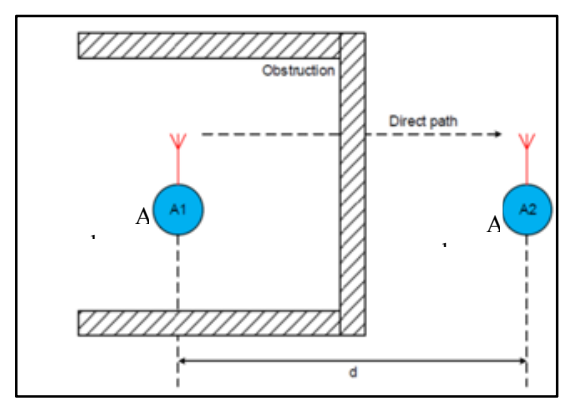

Fig. 4. NON Line OF Sight CHANNEL FROM [7]

\section{METHODOLOGY}

The basic methodology used in this article has two approaches: the mathematical expression and the Matlab simulation. For two and three anchor nodes accuracy compression, the computation of $\mathrm{x}$ and $\mathrm{y}$ coordinates for mobile position is made according to the algorithms below:

\section{A. Mathematical expression}

Positioning calculation for mobile station (MS) with 3 or more anchor nodes. Usually to find a position of a mobile station according to three anchor nodes or more, there are several algorithms such as least square method (LS), finger print, maximum likelihood estimate, etc. In this work, the implementation was by using least square method to build the positioning system. LS is a nonlinear method and needs for an iteration technique to compute the positioning of MS, but this method will be more complex in mathematical expression and costly in implementation time. Therefore, we linearized this problem by subtracting the first equation from the others as illustrated below

$$
\begin{gathered}
\text { Anchor nodes } x_{1}=y_{1}=0 \\
, x_{2}, y_{2}, x_{3}, y_{3}
\end{gathered}
$$

and $x_{n}, y_{n}$ are the anchor nodes coordinates

$$
\begin{gathered}
r_{1}^{2}=\left(x-x_{1}\right)^{2}+\left(y-y_{1}\right)^{2} \\
r_{2}^{2}=\left(x-x_{2}\right)^{2}+\left(y-y_{2}\right)^{2} \\
r_{3}^{2}=\left(x-x_{3}\right)^{2}+\left(y-y_{3}\right)^{2} \\
\vdots \\
r_{n}^{2}=\left(x-x_{n}\right)^{2}+\left(y-y_{n}\right)^{2}
\end{gathered}
$$

Using each equation to subtract the subsequent one we will get

$$
\left\{\begin{array}{c}
x_{1}^{2}-x_{n}^{2}-2\left(x_{1}-x_{n}\right) x+y_{1}^{2}-y_{n}^{2}-2\left(y_{1}-y_{n}\right) y=r_{1}^{2}-r_{n}^{2} \\
\vdots \\
x_{n-1}^{2}-x_{n}^{2}-2\left(x_{n-1}-x_{n}\right) x+y_{n-1}^{2}-y_{n}^{2}-2\left(y_{n-1}-y_{n}\right) y=r_{n-1}^{2}-r_{n}^{2}
\end{array}\right\}
$$




$$
\begin{gathered}
b=\left[\begin{array}{c}
x_{2}^{2}+y_{2}^{2}+r_{1}^{2}-r_{2}^{2} \\
x_{3}^{2}+y_{3}^{2}+r_{1}^{2}-r_{3}^{2} \\
\vdots \\
x_{n}^{2}+y_{n}^{2}+r_{1}^{2}-r_{n}^{2}
\end{array}\right] \\
\hat{b}=\left[\begin{array}{c}
x_{2}^{2}+y_{2}^{2}+d_{1}^{2}-d_{2}^{2} \\
x_{3}^{2}+y_{3}^{2}+d_{1}^{2}-d_{3}^{2} \\
\vdots \\
x_{n}^{2}+y_{n}^{2}+d_{1}^{2}-d_{n}^{2}
\end{array}\right] \\
d=r+e
\end{gathered}
$$

Where $e$ expresses the error in distance.

$$
\begin{aligned}
A & =\left[\begin{array}{cc}
x_{2} & y_{2} \\
x_{3} & y_{3} \\
\vdots & \vdots \\
x_{n} & y_{n}
\end{array}\right] \\
X & =\left[\begin{array}{l}
x \\
y
\end{array}\right]=\left(A^{T} A\right)^{-1} A^{T} b \\
\hat{X} & =\left[\begin{array}{l}
x \\
y
\end{array}\right]=\left(A^{T} A\right)^{-1} A^{T} \widehat{b}
\end{aligned}
$$

$r, d, b, \hat{b}, X, \widehat{X}$ denote real distance, estimated distance, distance vector, estimated distance vector, mobile position, and estimated mobile position, respectively.

Positioning calculation for mobile station with 2 anchor nodes.

In this scenario, we have to install the anchor nodes in a way that the both have the same $\mathrm{x}$ or $\mathrm{y}$ axis. To eliminate the ambiguity situation, we assumed the movement of the mobile station will be in front of us.

$$
\begin{gathered}
\text { Let } y_{1}=y_{2} \\
r_{1}^{2}=\left(x-x_{1}\right)^{2}+\left(y-y_{1}\right)^{2} \\
r_{2}^{2}=\left(x-x_{2}\right)^{2}+\left(y-y_{2}\right)^{2} \quad(4.14) \\
x_{1}^{2}-2 x x_{1}+x^{2}+y^{2}-2 y y_{1}+y_{1}^{2}=r_{1}^{2} \\
x_{2}^{2}-2 x x_{2}+x^{2}+y^{2}-2 y y_{2}+y_{2}^{2}=r_{2}^{2}
\end{gathered}
$$

By subtracting (1) from (2)

$$
\begin{array}{r}
r_{1}^{2}-r_{2}^{2}=x_{1}^{2}-x_{2}^{2}-2 x x_{1}+2 x x_{2} \\
r_{1}^{2}-r_{2}^{2}-x_{1}^{2}+x_{2}^{2}=2 x\left(x_{2}-x_{1}\right) \\
\hat{x}=\frac{r_{1}^{2}-r_{2}^{2}+x_{2}^{2}-x_{1}^{2}}{2 x\left(x_{2}-x_{1}\right)}
\end{array}
$$

From the equation (4.14) 


$$
\begin{gathered}
r_{2}^{2}=\left(x-x_{2}\right)^{2}+\left(y-y_{2}\right)^{2} \\
\left(y-y_{2}\right)^{2}=r_{2}^{2}-\left(x-x_{2}\right)^{2} \\
y-y_{2}=\mp \sqrt{r_{2}^{2}-\left(x_{2}-x\right)^{2}} \\
\hat{y}=y_{2} \mp \sqrt{r_{2}^{2}-\left(x_{2}-x\right)^{2}}
\end{gathered}
$$

$\widehat{x}$ and $\hat{y}$ are the coordinates of the estimated position.

\section{B.MATLAB simulation}

In Matlab simulation, we created a movement of the MS and then created LS method to locate the position of this mobile with two and three anchor nodes. Where the MS represented the mobile station, A1, A2, A3 represented the three anchor nodes and the R1, R2 represented the distance between the mobile node and the anchor node as shown in figure (7) where the position of anchor nodes are as the following:
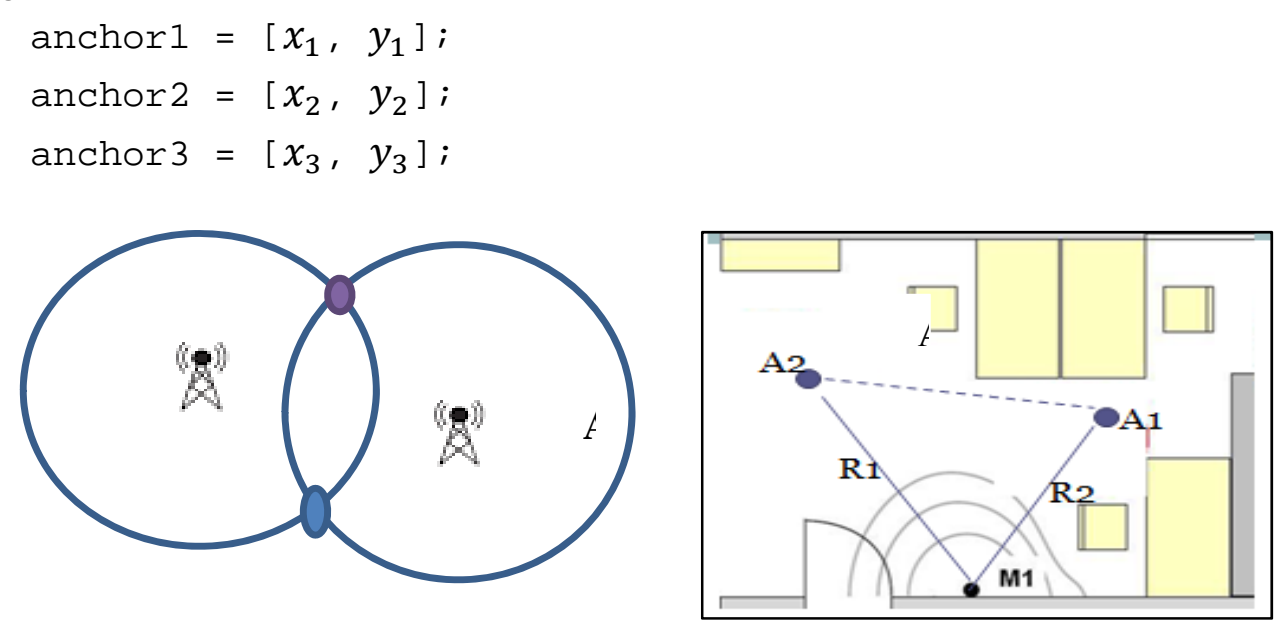

Fig. 5. REPRESENTATION OF THE DISTANCE ALGORITHM FOR 2 ANCHOR NODES.

The program will start to add different random noise (using rand function) (from $0.1 \mathrm{~m}$ to $0.2 \mathrm{~m}$ ) with one thousand estimated positions for each movement point. We computed the mean accumulation error for both scenarios with different values of error to compare the accuracy of two and three anchors in different movement situations of the MS. Figures 8, 9, 10,11, and Table 1 illustrate the aforementioned scenarios as shown in the following flowchart.

$$
\text { mean }_{\text {error }}=\frac{1}{n} \sum \hat{X}-X
$$

$n$ denotes the number of estimated positions. 


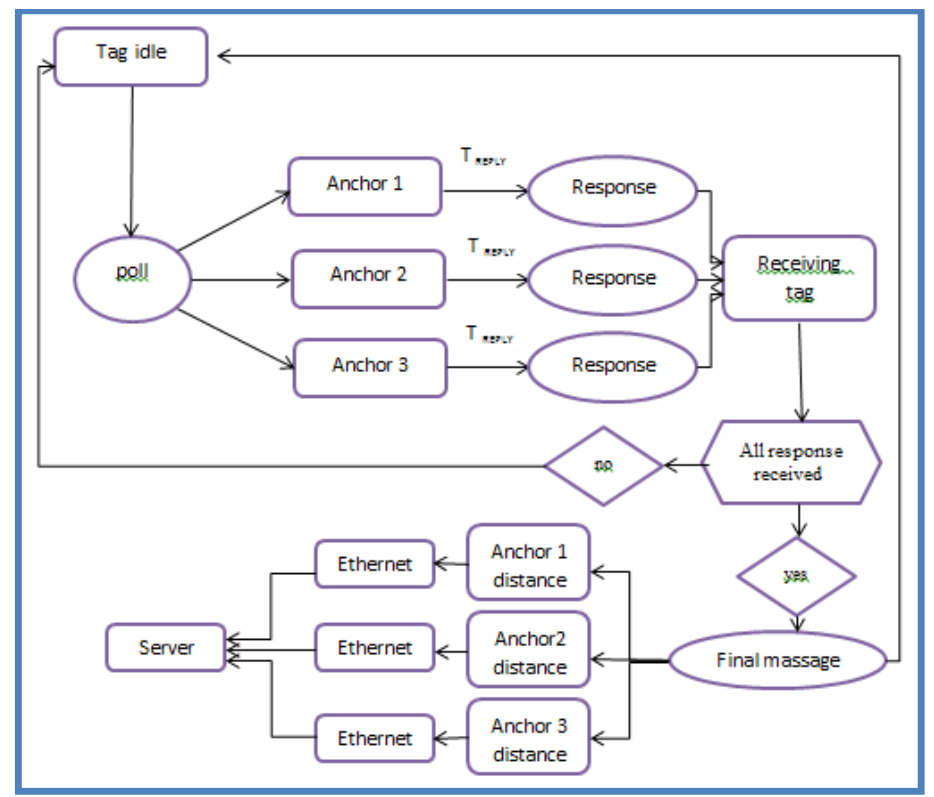

FIG. 6. FLOWCHART DESCRIBING THE POSITIONING OF ONE TAG

Table (1) represents the location of three anchor nodes and the mobile station with three different values of error in two situations, which are added randomly by the Matlab program code to find the mean position error for two and three anchor nodes, which prove that it is not always the case that three nodes could be a better position than two nodes and vice versa.

TABLE (1) THE LOCATION OF ANCHOR NODE WITH DIFFERENT VALUES OF ERROR

\begin{tabular}{|c|c|c|c|c|c|c|c|c|c|c|}
\hline \multirow[t]{2}{*}{$\begin{array}{l}\text { Num of } \\
\text { Anchor }\end{array}$} & \multirow{2}{*}{$\begin{array}{l}\text { Mean } \\
\text { position } \\
\text { error }\end{array}$} & \multirow[t]{2}{*}{ node } & \multirow{2}{*}{$\mathbf{x}$} & \multirow{2}{*}{ Y } & \multicolumn{2}{|c|}{$\begin{array}{l}\text { Mobile } \\
\text { station }\end{array}$} & \multicolumn{3}{|c|}{ error } & \multirow[t]{2}{*}{ Figure no } \\
\hline & & & & & $x$ & $y$ & E1 & E2 & E2 & \\
\hline \multirow{2}{*}{$\begin{array}{l}\text { Two } \\
\text { Anchor }\end{array}$} & 0.107 & A1 & 0 & 0 & 25 & -4 & 0.1 & 0.01 & 0.03 & Fig.9(a) \\
\hline & 1.682 & $\mathrm{~A} 2$ & 10 & 0 & 20 & -6 & 0.1 & 3 & 2 & Fig.10(a) \\
\hline \multirow{2}{*}{$\begin{array}{l}\text { Three } \\
\text { Anchor }\end{array}$} & 0.029 & \multirow[t]{2}{*}{$\mathrm{A} 3$} & \multirow[t]{2}{*}{10} & \multirow[t]{2}{*}{-10} & \multirow[t]{2}{*}{15} & \multirow[t]{2}{*}{-9} & 0.1 & 0.01 & 0.03 & Fig.9(b) \\
\hline & 0.571 & & & & & & 0.1 & 3 & 2 & Fig 10(b) \\
\hline
\end{tabular}

Figures (7-8) show that the real and the estimated mobile position of two anchor nodes is better than three anchor nodes, while figures (8-9) show that the mobile position with mean position error of three nodes is better than two anchor nodes with mean position error. 


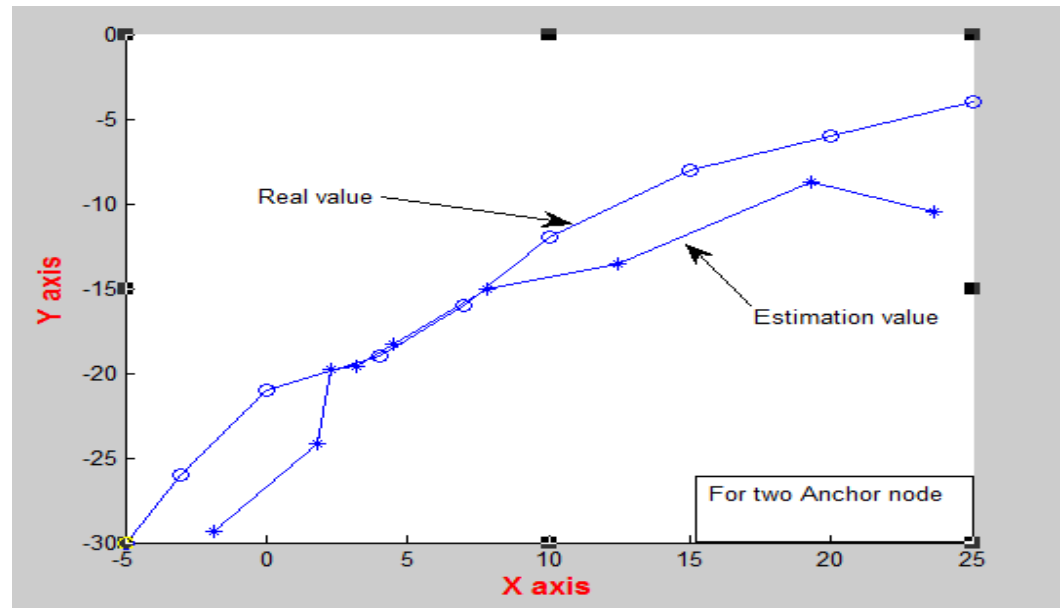

Fig. 7. REAL AND ESTIMATED VALUE OF MOBILE FOR 2 NODES

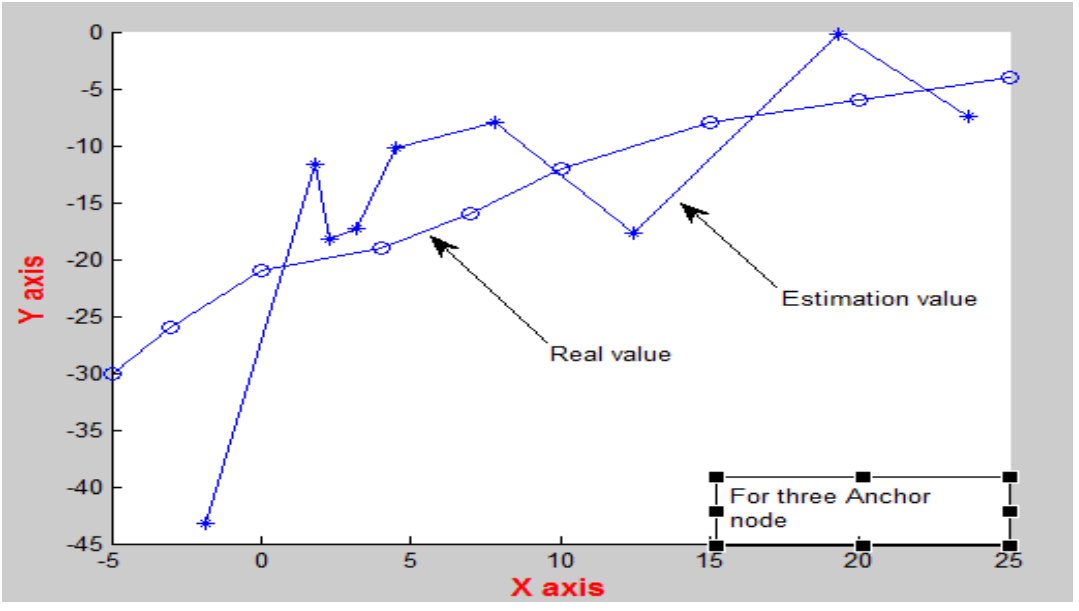

Fig. 8. REAL AND ESTIMATED VALUE OF MOBILE FOR 3 NODES

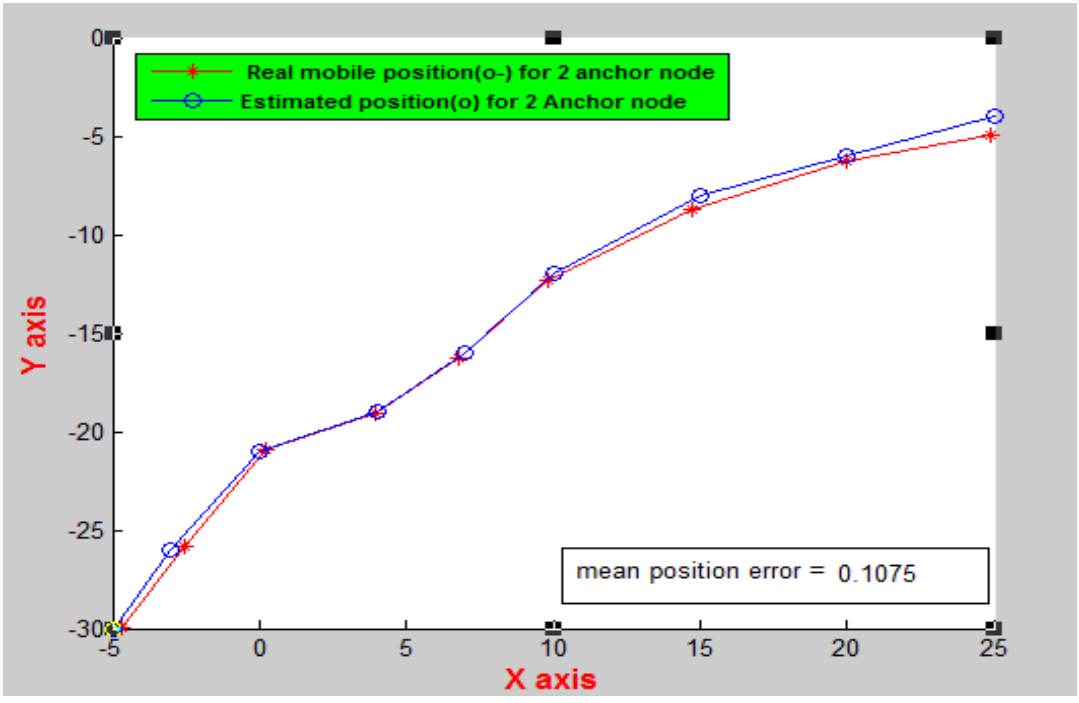

FIG. 9. (A) REAL AND ESTIMATED MOBILE POSITION WITH ERRORA FOR 2 NODES 


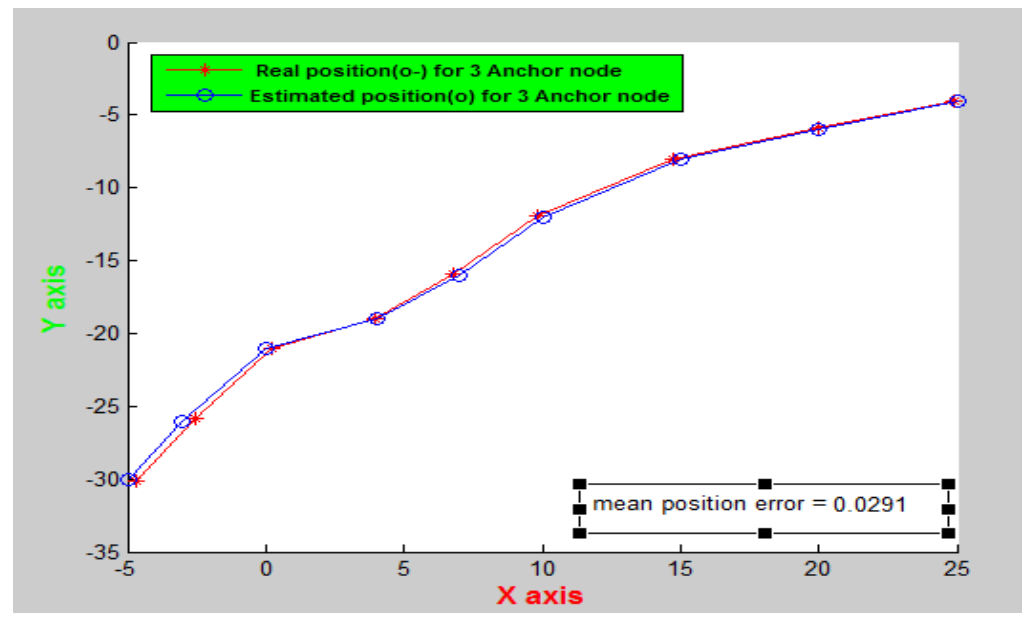

FIG. 9. (B) REAL AND ESTIMATED MOBILE POSITION WITH ERRORA FOR 3 NODES

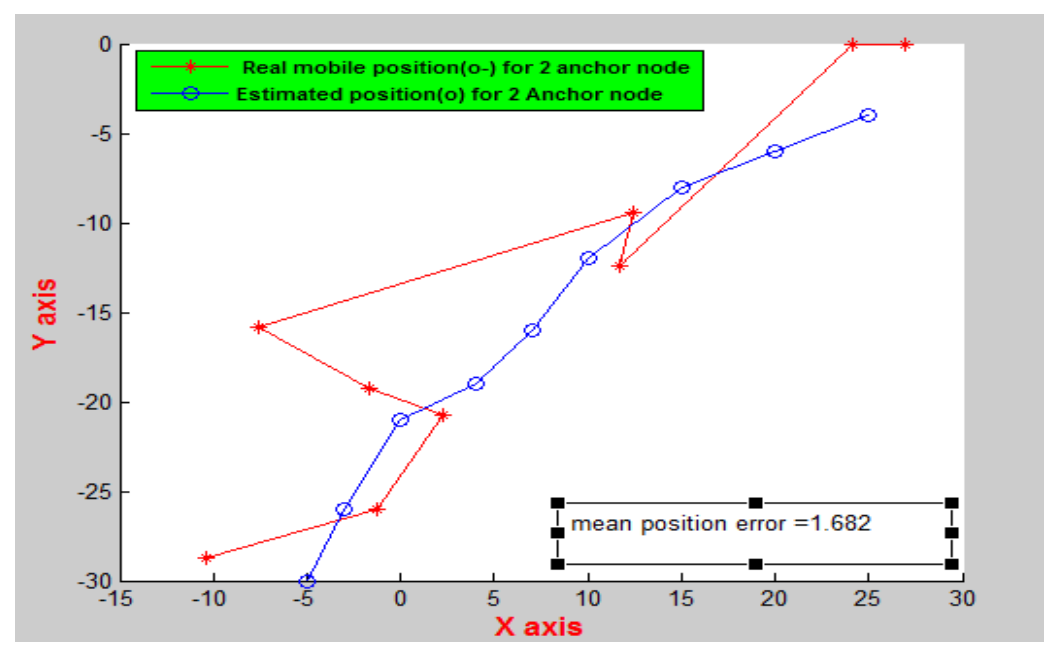

Fig. 10. (A) REAL AND ESTIMATED MOBILE POSITION WITH ERRORQFOR 2 NODES

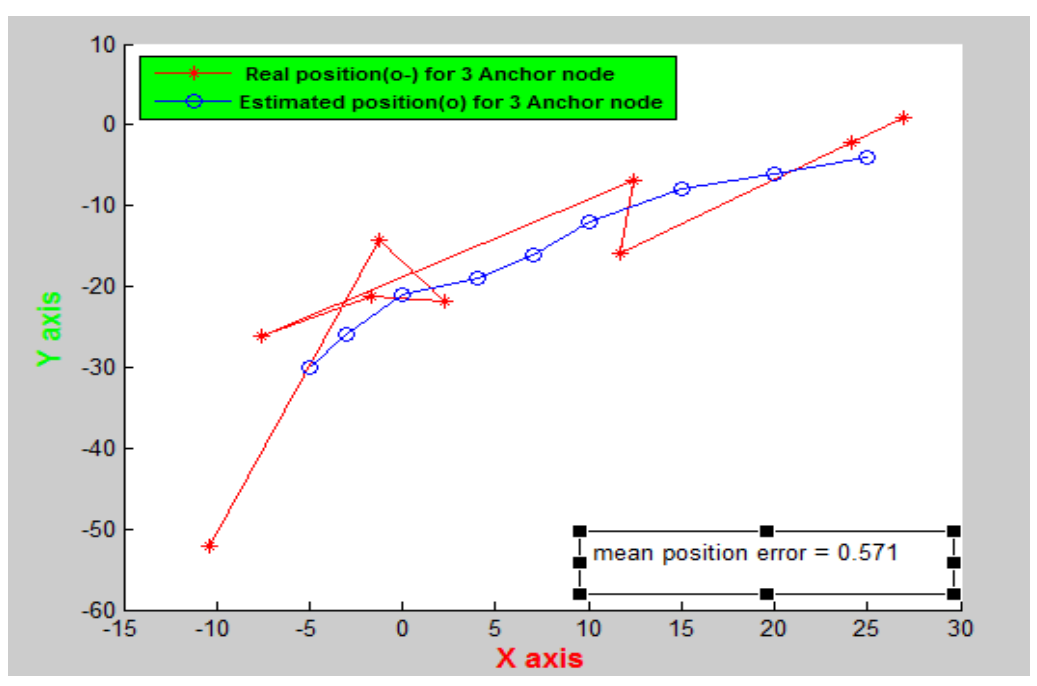

Fig.10 (B) REAL AND ESTIMATED MOBILE POSITION WITH ERRORQ FOR 3 NODES 


\section{CONCLUSION}

We have presented an indoor localization system, which is intended to have an accuracy of \pm 1 meter. The major advantage is the ability to locate any mobile device without additional hardware in the mobile and without any changes in its software. The local position is determined by differential time difference of arrival measurements. We proved that it is no not always the case that having a larger number of anchor nodes will increase the accuracy. Two and three anchor nodes of ultra-wide band commercial devices (DW 1000) could be implemented in the next work to build the IPS and the results showed that sometimes three anchor nodes are better than two and vice versa. A reliable, precise and real-time IPS has been created in this work. It depends on how to install the anchor nodes in an appropriate scenario that may allow utilizing a smaller number of anchors while maintaining the required accuracy and cost. Due to the differential nature of the time measurements, there is no need to synchronize the clocks of the involved measurement stations. The time of arrival measurements require in essence line-of-sight conditions for the indoor radio channel.

\section{REFERANCES}

[1] G. Oliva, S. Panzieri, F. Pascucci, R. Setola, Network localization by shadow edges, in: 12th European Control Conference, ECC2013, Zurich, Switzerland, 17-19 July 2013, pp. 2263-2268.

[2] G. Oliva, S. Panzieri, F. Pascucci, R. Setola, Sensor network localization: Extending trilateration via shadow edges, IEEE Trans. Automat. Control 60 (10) (2015) 2752-2755.

[3] K.C. Ho, W. Xu, An accurate algebraic solution for moving source location using TDOA and FDOA measurement, IEEE Trans. Signal Process. 52(9)(2004) 2453-2463.

[4] Yayan, U. Yucel, H. Yazici, A. 2014. A Low Cost Ultrasonic Based Position-ing System for the Indoor Navigation of Mobile Robots. Journal of Intelligent \& Robotic Systems.

[5] H. Kim, Double-sided two-way ranging algorithm to reduce ranging time, IEEE Commun. Lett. 13.

[6] R. Price and P. E. Green, Jr., “A Communication Technique for Multipath Channels”, Proc. IRE,Vol. 46, pp. 555-570 (March 1958)

[7] Decawave Ltd.APS006 Application Note: channel effects on communications range and time stamp accuracy, http//www.decawave.com/, 2014. 